\title{
Rebound Distinguishers: Results on the Full Whirlpool Compression Function
}

\author{
Mario Lamberger ${ }^{1}$, Florian Mendel $^{1}$, Christian Rechberger $^{1}$, \\ Vincent Rijmen ${ }^{1,2,3}$, and Martin Schläffer ${ }^{1}$ \\ 1 Institute for Applied Information Processing and Communications \\ Graz University of Technology, Inffeldgasse 16a, A-8010 Graz, Austria \\ 2 Department of Electrical Engineering ESAT/COSIC, Katholieke Universiteit \\ Leuven. Kasteelpark Arenberg 10, B-3001 Heverlee, Belgium \\ 3 Interdisciplinary Institute for BroadBand Technology (IBBT), Belgium \\ mario.lamberger@iaik. tugraz.at
}

\begin{abstract}
Whirlpool is a hash function based on a block cipher that can be seen as a scaled up variant of the AES. The main difference is the (compared to AES) extremely conservative key schedule. In this work, we present a distinguishing attack on the full compression function of Whirlpool. We obtain this result by improving the rebound attack on reduced Whirlpool with two new techniques. First, the inbound phase of the rebound attack is extended by up to two rounds using the available degrees of freedom of the key schedule. This results in a near-collision attack on 9.5 rounds of the compression function of Whirlpool with a complexity of $2^{176}$ and negligible memory requirements. Second, we show how to turn this near-collision attack into a distinguishing attack for the full 10 round compression function of Whirlpool. This is the first result on the full Whirlpool compression function.
\end{abstract}

Keywords: hash functions, cryptanalysis, near-collision, distinguisher.

\section{Introduction}

In the last few years the cryptanalysis of hash functions has become an important topic within the cryptographic community. Especially the collision attacks on the MD4 family of hash functions (MD4, MD5, SHA-1) have weakened the security assumptions of these commonly used hash functions [6/7/17/24/25/26]. Still, most of the existing cryptanalytic work has been published for this particular family of hash functions. Therefore, the analysis of alternative hash functions is of great interest. In this article, we will present a security analysis of the Whirlpool hash function with respect to collision resistance.

Whirlpool is the only hash function standardized by ISO/IEC 10118-3:2004 (since 2000) that does not follow the MD4 design strategy. Furthermore, it has been evaluated and approved by NESSIE [20]. Whirlpool is commonly considered to be a conservative block-cipher based design with an extremely conservative key schedule and follows the wide-trail design strategy 4/5. Since its proposal in 2000, only a few results have been published. 
Table 1. Summary of results for Whirlpool. Complexities are given in compression function evaluations, a memory unit refers to a state (512 bits). The complexities in brackets refer to modified attacks using a precomputed table taking $2^{128}$ time/memory to set up.

\begin{tabular}{|c|c|c|c|c|}
\hline target & rounds & $\begin{array}{c}\text { complexity } \\
\text { runtime/memory }\end{array}$ & type & source \\
\hline block cipher $W$ & 6 & $2^{120} / 2^{120}$ & distinguisher & "Knudsen [11 \\
\hline $\begin{array}{c}\text { hash function } \\
\text { hash function } \\
\text { compression function } \\
\text { compression function }\end{array}$ & $\begin{array}{l}4.5 \\
6.5 \\
5.5 \\
7.5\end{array}$ & $\begin{array}{l}2^{120} / 2^{7} \\
2^{128} / 2^{7} \\
2^{120} / 2^{7} \\
2^{128} / 2^{7}\end{array}$ & $\begin{array}{c}\text { collision } \\
\text { near-collision } \\
\text { collision } \\
\text { near-collision }\end{array}$ & $\begin{array}{l}\text { Mendel et al. } \\
\text { FSE } 2009[16\end{array}$ \\
\hline $\begin{array}{c}\text { hash function } \\
\text { hash function } \\
\text { compression function } \\
\text { compression function } \\
\text { compression function }\end{array}$ & $\begin{array}{l}5.5 \\
7.5 \\
7.5 \\
9.5 \\
10\end{array}$ & $\begin{array}{c}2^{120+s} / 2^{64-s} \\
2^{128+s} / 2^{64-s} \\
2^{184} / 2^{8}\left(2^{120} / 2^{128}\right) \\
2^{176} / 2^{8}\left(2^{112} / 2^{128}\right) \\
2^{188} / 2^{8}\left(2^{121} / 2^{128}\right)\end{array}$ & $\begin{array}{c}\text { collision } \\
\text { near-collision } \\
\text { collision } \\
\text { near-collision } \\
\text { distinguisher }\end{array}$ & $\begin{array}{l}\text { Appendix } \mathrm{A} \\
\text { Appendix } \mathrm{A} \\
\text { Sect. } 4 \\
\text { Sect. } 4 \\
\text { Sect. } 5\end{array}$ \\
\hline
\end{tabular}

Related Work. At FSE 2009, Mendel et al. proposed a new technique for the analysis of hash functions: the rebound attack [16. It can be applied to both block cipher based and permutation based constructions. The idea of the rebound attack is to divide an attack into two phases, an inbound and an outbound phase. In the inbound phase, degrees of freedom are used, such that in the outbound phase several rounds can be bypassed in both forward- and backwards direction. This led to successful attacks on round-reduced Whirlpool for up to 7.5 (out of 10) rounds. The results are summarized in Table 1

For the block cipher $W$ that is implicitly used in the Whirlpool compression function, Knudsen described an integral distinguisher for 6 out of 10 rounds [1]. Furthermore, it is assumed that this property may extend also to 7 rounds. Note that in 12 similar techniques were used to obtain known-key distinguishers for 7-rounds of the AES.

Our Contribution. The main contribution of this paper is a distinguishing attack on the full compression function of Whirlpool which is achieved by improving upon the work of Mendel et al. in [16] in several ways.

We start with a description of the hash function Whirlpool. Then, in Sect. 3. we give an overview of the rebound attack and show how it is applied to reduced versions of Whirlpool. In Sect. 4, we describe our improvement of the rebound attack on Whirlpool in detail. This technique enables us to add two rounds in the inbound phase of the attack and thus gives a collision and near-collision attack on the Whirlpool compression function reduced to 7.5 and 9.5 rounds, respectively. Based on this, we describe in Sect. 5 a new generic attack and show how to distinguish the full (all 10 rounds) compression function of Whirlpool from a random function by turning the near-collision attack for 9.5 rounds into a distinguishing attack for 10 rounds. To the best of our knowledge this is the first result on the full Whirlpool compression function. Table 1 summarizes the previous results on Whirlpool as well as the contributions of this paper. 


\section{Description of Whirlpool}

Whirlpool is a cryptographic hash function designed by Barreto and Rijmen in 2000 [1. It is an iterative hash function based on the Merkle-Damgård design principle ( $c f$. 18]). It processes 512-bit message blocks and produces a 512-bit hash value. If the message length is not a multiple of 512, an unambiguous padding method is applied. For the description of the padding method we refer to [1]. Let $M=M_{1}\left\|M_{2}\right\| \cdots \| M_{t}$ be a t-block message (after padding). The hash value $h=H(M)$ is computed as follows:

$$
\begin{aligned}
H_{0} & =I V \\
H_{j} & =W\left(H_{j-1}, M_{j}\right) \oplus H_{j-1} \oplus M_{j} \quad \text { for } 0<j \leq t \\
h & =H_{t}
\end{aligned}
$$

where $I V$ is a predefined initial value and $W$ is a 512 bit block cipher used in the Miyaguchi-Preneel mode [18]. The block cipher $W$ used by Whirlpool is very similar to the Advanced Encryption Standard (AES) [19].

The state update transformation and the key schedule update an $8 \times 8$ state $S$ and $K$ of 64 bytes in 10 rounds. In one round, the state is updated by the round transformation $r_{i}$ as follows:

$$
r_{i} \equiv \mathrm{AK} \circ \mathrm{MR} \circ \mathrm{SC} \circ \mathrm{SB} .
$$

The round transformations are briefly described here:

- the non-linear layer SubBytes (SB) applies an S-Box to each byte of the state independently.

- the cyclical permutation ShiftColumns (SC) rotates the bytes of column $j$ downwards by $j$ positions.

- the linear diffusion layer MixRows (MR) is a right-multiplication by the $8 \times 8$ circulant MDS matrix $\operatorname{cir}(1,1,4,1,8,5,2,9)$.

- the key addition AddRoundKey (AK) adds the round key $K_{i}$ to the $8 \times 8$ state, and AddConstant $(\mathrm{AC})$ adds the round constant $C_{i}$ to the $8 \times 8$ state of the key schedule.

After the last round of the state update transformation, the initial value or previous chaining value $H_{j-1}$, the message block $M_{j}$, and the output value of the last round are combined (xored), resulting in the output of one iteration. A detailed description of the hash function is given in [1].

We denote the resulting state of round transformation $r_{i}$ by $S_{i}$ and the intermediate states after SubBytes by $S_{i}^{\mathrm{SB}}$, after ShiftColumns by $S_{i}^{\mathrm{SC}}$ and after MixRows by $S_{i}^{\mathrm{MR}}$. The initial state prior to the first round is denoted by $S_{0}=M_{j} \oplus K_{0}$. The same notation is used for the key schedule with round keys $K_{i}$ with $K_{0}=H_{j-1}$.

\section{The Rebound Attack}

The rebound attack is a new tool for the cryptanalysis of hash functions and was published by Mendel et al. in [16. It is a differential attack. The main 
idea is to use the available degrees of freedom in a collision attack to efficiently fulfill the low probability parts in the middle of a differential trail. The rebound attack consists of an inbound phase with a meet-in-the-middle part in order to exploit the available degrees of freedom, and a subsequent probabilistic outbound phase. AES based hash functions are a natural target for this attack, since their construction principle allows a simple application of the idea.

\subsection{Basic Attack Strategy}

In the rebound attack, the compression function, internal block cipher or permutation of a hash function is split into three sub-parts. Let $W$ be a block cipher, then $W=W_{f w} \circ W_{i n} \circ W_{b w}$.

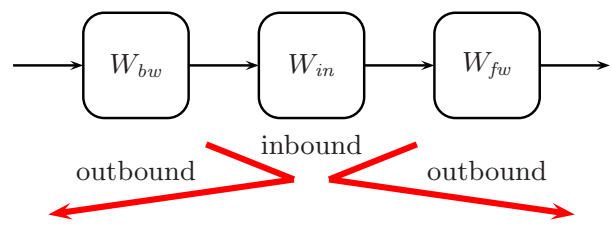

Fig. 1. A schematic view of the rebound attack. The attack consists of an inbound and two outbound phases.

The rebound attack can be described by two phases (see Fig. 1):

- Inbound phase: Is a meet-in-the-middle phase in $W_{i n}$, which is aided by the degrees of freedom that are available to a hash function cryptanalyst. This very efficient combination of meet-in-the-middle techniques with the exploitation of available degrees of freedom is called the match-in-themiddle approach.

- Outbound phase: In the second phase, the matches of the inbound phase are computed in both forward- and backward direction through $W_{f w}$ and $W_{b w}$ to obtain desired collisions or near-collisions. If the differential trail through $W_{f w}$ and $W_{b w}$ has a low probability, one has to repeat the inbound phase to obtain more starting points for the outbound phase.

\subsection{Preliminaries for the Rebound Attack on Whirlpool}

In the following, we want to briefly summarize some well known facts that will be frequently used in the subsequent sections.

- Truncated differentials: Knudsen [10] proposed truncated differentials as a tool in block cipher cryptanalysis. In a standard differential attack ( $c f$. [2]), the full difference between two inputs/outputs is considered whereas in the case of truncated differentials, the differences is only partially determined, i.e. for every byte, we only check if there is a difference or not. A byte having a non-zero difference is called active. 
- Difference Propagation in MixRows: Since the MixRows operation is a linear transformation, standard differences propagate through MixRows in a deterministic way whereas truncated differences behave in a probabilistic way. The MDS property of the MixRows transformation ensures that the sum of the number of active input and output bytes is at least 9 (cf. 1]). In general, the probability of any $x \rightarrow y$ transition with $1 \leq x, y \leq 8$ satisfying $x+y \geq 9$ is approximately $2^{(y-8) \cdot 8}$. For a detailed description of the propagation of truncated differences in MixRows we refer to [16, see also 21.

- Differential Properties of SubBytes: Let $a, b \in\{0,1\}^{8}$. For the Whirlpool S-box, we are interested in the number of solutions to the equation

$$
S(x) \oplus S(x \oplus a)=b .
$$

Exhaustively counting over all $2^{16}$ differentials shows that the number of solutions to (4) can only be $0,2,4,6,8$ and 256, which occur with frequency $39655,20018,5043,740,79$ and 1 , respectively. The task to return all solutions $x$ to (4) for a given differential $(a, b)$ is best solved by setting up a precomputed table of size $256 \times 256$ which stores the solutions (if there are any) for each $(a, b)$.

However, it is easy to see that for any permutation $S$ (to be more precise, for any injective map) the expected number of solutions to (4) is always 1. We get that $2^{-16} \sum_{a} \sum_{b} \#\{x \mid S(x \oplus a) \oplus S(x)=b\}=2^{-16} \sum_{a} 2^{8}=1$, because for a fixed $a$, every solution $x$ belongs to a unique $b$. Since the inputs to all the S-boxes are independent, the same reasoning is valid for the full SubBytes transformation.

\subsection{Application to Round-Reduced Whirlpool}

In this section, we will briefly describe the application of the rebound attack to the hash function Whirlpool. A detailed description of the attack is given in [16]. For a good understanding of our results, it is recommended to study these previous results on Whirlpool very carefully.

The rebound attack on Whirlpool is a differential attack which uses a differential trail with the minimum number of active S-boxes according to the wide trail design strategy. The core of the rebound attack on Whirlpool is a 4 round differential trail, where the fully active state is placed in the middle:

$$
1 \stackrel{r_{1}}{\longrightarrow} 8 \stackrel{r_{2}}{\longrightarrow} 64 \stackrel{r_{3}}{\longrightarrow} 8 \stackrel{r_{4}}{\longrightarrow} 1
$$

In the rebound attack, one first splits the block cipher $W$ into three sub-ciphers $W=W_{f w} \circ W_{i n} \circ W_{b w}$, such that the most expensive part of the differential trail is covered by the inbound phase $W_{i n}$. In the inbound phase, the available degrees of freedom (in terms of actual values of the state) are used to guarantee that the differential trail in $W_{i n}$ holds. The differential trail in the outbound phase $\left(W_{f w}, W_{b w}\right)$ is supposed to have a relatively high probability. While standard XOR differences are used in the inbound phase, truncated differentials are used in the outbound phase of the attack. 


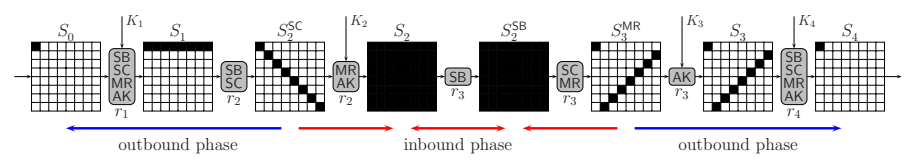

Fig. 2. A schematic view of the rebound attack on 4 rounds of Whirlpool with round key inputs. Black state bytes are active.

In the following, we briefly describe the inbound and outbound phase of the rebound attack on 4 rounds of Whirlpool. For a more detailed description, we refer to the original paper [16].

Inbound Phase. In the first step of the inbound phase, we choose a random difference with 8 active bytes at the input of MixRows of round $r_{2}\left(S_{2}^{\mathrm{SC}}\right)$. Note that we need an active byte in each row of the state (see Fig. 2) to get a fully active state after the MixRows transformation. Since AddRoundKey does not change the difference, we get a fully active state at the input of SubBytes of round $r_{3}\left(S_{2}\right)$. Then, we start with another difference in 8 active bytes at the output of MixRows of round $r_{3}\left(S_{3}^{\mathrm{MR}}\right)$ and propagate backwards. Again, since we have an active byte in each row, we get a fully active state at the output of SubBytes of round $r_{3}$.

In the second step of the inbound phase, the match-in-the-middle step, we look for a matching input/output difference of the SubBytes layer of round $r_{3}$. This is done as described in Sect. 3.2 with a precomputed $256 \times 256$ lookup table. Note that we can repeat the inbound phase at most about $2^{128}$ times. As indicated in Sect. 3.2, we expect one solution per trial, that is, we can produce at most $2^{128}$ actual values that follow the differential trail in the inbound phase.

Outbound Phase. In contrast to the inbound phase, we use truncated differentials in the outbound phase of the attack. By propagating the matching differences and state values through the next SubBytes layer outwards, we get a truncated differential in 8 active bytes in both backward and forward direction. These truncated differentials need to propagate from 8 to 1 active byte through the MixRows transformation, both in the backward and forward direction (see Fig. 2). The propagation of truncated differentials through the MixRows transformation can be modelled in a probabilistic way, see Sect. 3.2. Since we need to fulfill one $8 \rightarrow 1$ transitions in the backward and forward direction, the probability of the outbound phase is $2^{-2 \cdot 56}=2^{-112}$. In other words, we have to repeat the inbound phase about $2^{112}$ times to generate $2^{112}$ starting points for the outbound phase of the attack.

\subsection{Previous Results on Round-Reduced Whirlpool}

Extending the 4 round trail in both, the inbound and outbound phase, leads to attacks on round reduced Whirlpool for up to 7.5 (out of 10) rounds (where 0.5 rounds consist only of SubBytes and ShiftColumns). To be more precise, by 
extending the outbound phase of the attack by 0.5 and 2.5 rounds, one can construct a collision and near-collision for the Whirlpool hash function reduced to 4.5 and 6.5 rounds, respectively. The collision attack has a complexity of about $2^{120}$ and the near-collision attack has a complexity of about $2^{128}$. Furthermore, by additionally extending the inbound phase of the attack by 1 round, one can find a collision and a near-collision for the compression function of Whirlpool reduced to 5.5 and 7.5 rounds with a complexity of $2^{120}$ and $2^{128}$, respectively. Note that adding this round in the inbound phase is possible, since in a compression function attack, one can use the degrees of freedom of the key schedule (chaining value) to guarantee that the trail in the inbound phase holds. All results are summarized in Table 1 and for more details on these results we refer to 16 .

\section{Improved Rebound Attack on the Whirlpool Compression Function}

In this section, we improve the inbound phase of the original rebound attack on Whirlpool. By using a new differential trail and extensively using the available degrees of freedom of the key schedule, we can add 2 additional rounds to the inbound phase of the attacks. The basic idea is to have two instead of one inbound phase (match-in-the-middle step) and connect them using the available degrees of freedom from the key schedule. The outbound phase of the attacks is identical as in the previous attacks on 5.5 and 7.5 rounds for the compression function of Whirlpool. As a result, we obtain a collision and a near-collision attack for the compression function of Whirlpool reduced 7.5 and 9.5 rounds, respectively.

\subsection{Inbound Phase}

In this section, we describe the improved inbound phase of the attack in detail. We use the following sequence of active bytes:

$$
8 \stackrel{r_{1}}{\longrightarrow} 64 \stackrel{r_{2}}{\longrightarrow} 8 \stackrel{r_{3}}{\longrightarrow} 8 \stackrel{r_{4}}{\longrightarrow} 64 \stackrel{r_{5}}{\longrightarrow} 8
$$

In order to find inputs following the differential of the inbound phase, we split it into two parts. In the first part, we apply the match-in-the-middle step with active bytes $8 \rightarrow 64 \rightarrow 8$ twice in rounds $1-2$ and $4-5$. In the second part, we need to connect the resulting 8 active bytes and 64 (byte) values of the state between round 2 and 4 using the degrees of freedom we have in the choice of the round key values (see Fig. 33).

Inbound Part 1. In this part of the inbound phase, we apply the match-in-themiddle step twice for rounds 1-2 and 4-5 (see Fig. 3), which can be summarized as follows:

1. Precomputation: For the S-box, compute a $256 \times 256$ lookup table as described in Sect. 3.2 . 


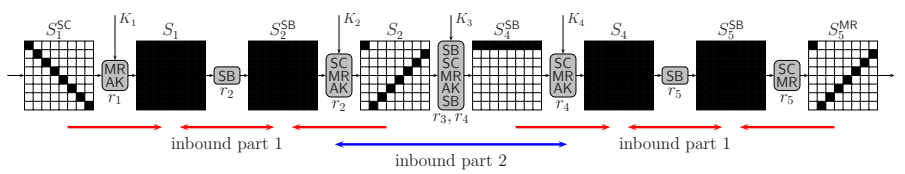

Fig. 3. The inbound phase of the attack

2. Match-in-the-middle (rounds 1-2):

(a) Start with 8 active bytes at the output of AddRoundKey in round $r_{2}\left(S_{2}\right)$ and propagate backward to the output of SubBytes in round $r_{2}\left(S_{2}^{\mathrm{SB}}\right)$.

(b) Start with 8 active bytes at the input of MixRows in round $r_{1}\left(S_{1}^{\mathrm{SC}}\right)$ and propagate forward to the input of SubBytes in round $r_{2}\left(S_{1}\right)$. Note that we can compute forward and solve the following step for each row independently.

(c) Connect the input and output of the S-boxes of round $r_{2}$ by choosing the actual values of the state $S_{1}$, respectively $S_{2}^{\mathrm{SB}}$, using the lookup table generated in the precomputation step. After repeating step (b) for each row about $2^{8}$ times we expect to find a match for the $8 \mathrm{~S}$ boxes and thus $2^{8}$ actual values (see Sect. 3.2). Since we do this for all rows independently, we get about $2^{64}$ actual values for the full state $S_{1}$, respectively $S_{2}^{\mathrm{SB}}$, such that the trail holds.

3. Match-in-the-middle (rounds 4-5): Do the same as in Step 2.

Hence, we get $2^{64}$ candidates for $S_{2}^{\mathrm{SB}}$ and $2^{64}$ candidates for $S_{4}$ after the first part of the inbound phase of the attack with a complexity of about $2^{9}$ round transformations.

Inbound Part 2. In the second part of the inbound phase, we have to connect the 8 active bytes (64 (bit) conditions) as well as the actual values (512 (bit) conditions) of $S_{2}^{\mathrm{SB}}$ and $S_{4}$ by choosing the subkeys $K_{2}, K_{3}$ and $K_{4}$ accordingly. Therefore, we have to solve the following equation:

$$
\operatorname{MR}\left(\mathrm{SC}\left(\mathrm{SB}\left(\mathrm{MR}\left(\mathrm{SC}\left(\mathrm{SB}\left(\mathrm{MR}\left(\mathrm{SC}\left(S_{2}^{\mathrm{SB}}\right)\right) \oplus K_{2}\right)\right)\right) \oplus K_{3}\right)\right)\right) \oplus K_{4}=S_{4}
$$

with

$$
\begin{aligned}
& K_{3}=\operatorname{MR}\left(\mathrm{SC}\left(\mathrm{SB}\left(K_{2}\right)\right)\right) \oplus C_{3} \\
& K_{4}=\operatorname{MR}\left(\operatorname{SC}\left(\mathrm{SB}\left(K_{3}\right)\right)\right) \oplus C_{4} .
\end{aligned}
$$

Since we have $2^{64}$ candidates for $S_{2}^{\mathrm{SB}}, 2^{64}$ candidates for $S_{4}$ and $2^{512}$ candidates for the 3 subkeys $K_{2}, K_{3}, K_{4}$ (because of (6) ), we expect to find $2^{64}$ solutions.

Since $S_{2}^{\mathrm{MR}}=\operatorname{MR}\left(\mathrm{SC}\left(S_{2}^{\mathrm{SB}}\right)\right)$, we can rewrite the above equation as follows:

$$
\operatorname{MR}\left(\operatorname{SC}\left(\operatorname{SB}\left(\operatorname{MR}\left(\operatorname{SC}\left(\operatorname{SB}\left(S_{2}^{\mathrm{MR}} \oplus K_{2}\right)\right)\right) \oplus K_{3}\right)\right)\right) \oplus K_{4}=S_{4}
$$

Note that one can always change the order of SC and SB in the Whirlpool block cipher without affecting the output of one round. In order to make the 
subsequent description of the attack easier, we do this here and get the following equation.

$$
\operatorname{MR}\left(\mathrm{SC}\left(\mathrm{SB}\left(\mathrm{MR}\left(\mathrm{SB}\left(\mathrm{SC}\left(S_{2}^{\mathrm{MR}} \oplus K_{2}\right)\right)\right) \oplus K_{3}\right)\right)\right) \oplus K_{4}=S_{4}
$$

Furthermore, MR and SC are linear transformations and hence we can rewrite the above equation as follows:

$$
\mathrm{SB}\left(\mathrm{MR}\left(\mathrm{SB}\left(S_{2}^{*} \oplus K_{2}^{*}\right)\right) \oplus K_{3}\right) \oplus K_{4}^{\mathrm{SB}}=X
$$

with $S_{2}^{*}=\mathrm{SC}\left(S_{2}^{\mathrm{MR}}\right), K_{2}^{*}=\mathrm{SC}\left(K_{2}\right), K_{4}^{\mathrm{SB}}=\mathrm{SB}\left(K_{3}\right), X=\mathrm{SC}^{-1}\left(\mathrm{MR}^{-1}\left(S_{4} \oplus C_{4}\right)\right)$.

In the following, this equivalent description is used to connect the values and differences of the two states $S_{2}^{\mathrm{MR}}$ and $S_{4}$.

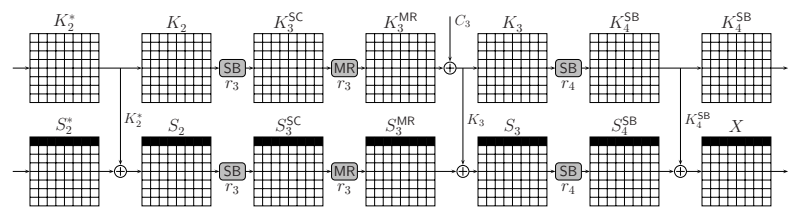

Fig. 4. The second part of the inbound phase. Black state bytes are active.

Remember that the two 8-byte differences of $S_{2}^{*}$ and $X$ have already been fixed due to the previous steps. Furthermore, we can choose from $2^{64}$ values for each of the states $S_{2}^{*}$ and $X$. Now, we use equation (9) to determine the subkey $K_{2}^{*}$ such that we get a solution for the inbound phase of the attack. Note that we can solve (9) for each row of the equation independently (see Fig. 44). It can be summarized as follows.

1. Compute the 8 -byte difference and the $2^{64}$ values of the state $S_{2}^{*}$ from $S_{2}^{\mathrm{SB}}$, and compute the 8 -byte difference and the $2^{64}$ values of the state $X$ from $S_{4}$. Note that we can compute and store the values of $S_{2}^{*}$ and $X$ row-by-row and independently. Hence, both the complexity and memory requirements for this step are $2^{8}$ instead of $2^{64}$.

2. Repeat the following steps for all $2^{64}$ values of the first row of $S_{2}$ to get $2^{64}$ matches for $S_{2}^{*}$ to $S_{4}$ :

(a) For the chosen value of the first row of $S_{2}$, forward compute the differences and values to the first row of $S_{3}$.

(b) Choose the first row of the key $K_{3}$ such that the differential of the S-box between $S_{3}$ and $S_{4}^{\mathrm{SB}}$ holds.

(c) Compute the first row of $K_{2}^{*}, S_{2}^{*}, K_{4}^{\mathrm{SB}}$ and $X$. Since we have $2^{64}$ values for the first row of $S_{2}^{*}$ and $2^{64}$ values for the first row of $X$, we expect to find a match on both sides. In other words, we have now connected the values and differences of the first row.

(d) Next, we connect the values of rows 2-8 independently by a simple bruteforce search over all $2^{64}$ corresponding key values of $K_{2}^{*}$. Since we have to connect 64 bit values and we test $2^{64}$ key values we expect to always find a solution. 
In total, we get $2^{64}$ matches connecting state $S_{2}^{*}$ to state $X$ with a complexity of $2^{128}$ and memory requirements of $2^{8}$. In other words, with the values of $S_{2}^{*}, X$ and the corresponding key $K_{2}^{*}$, we get $2^{64}$ starting points for the outbound phase of the attack. Hence, the average complexity to find one starting point for the outbound phase is $2^{64}$. It is important to note that one can construct a total of $2^{192}$ starting points in the inbound phase to be used in the outbound phase of the attack.

Note that step 2 (d) can be implemented using a precomputed lookup table of size $2^{128}$. In this lookup table each row of the key $K_{2}(64 \mathrm{bits})$ is saved for the corresponding two rows of $S_{2}^{*}$ and $X$ (64 bits each). Using this lookup table, we can find one starting point for the outbound phase with an average complexity of 1 . However, the complexity to generate this lookup table is $2^{128}$.

\subsection{Outbound Phase}

In the outbound phase of the attack, we further extend the differential path backward and forward. By propagating the matching differences and state values through the next SubBytes layer, we get a truncated differential in 8 active bytes for each direction. These truncated differentials need to follow a specific active byte pattern to result in a collision on 7.5 rounds and a near-collision on 9.5 rounds, respectively. In the following, we will describe the outbound phase for the collision and near-collision attack in detail.

Collision for 7.5 Rounds. By adding 1 round in the beginning and 1.5 rounds at the end of the trail, we get a collision for 7.5 rounds for the compression function of Whirlpool. In the attack, we use the following sequence of active bytes:

$$
1 \stackrel{r_{1}}{\longrightarrow} 8 \stackrel{r_{2}}{\longrightarrow} 64 \stackrel{r_{3}}{\longrightarrow} 8 \stackrel{r_{4}}{\longrightarrow} 8 \stackrel{r_{5}}{\longrightarrow} 64 \stackrel{r_{6}}{\longrightarrow} 8 \stackrel{r_{7}}{\longrightarrow} 1 \stackrel{r_{7.5}}{\longrightarrow} 1
$$

As described in Sect. 3.2, the propagation of truncated differentials through the MixRows transformation is modelled in a probabilistic way. For the differential trail to hold, we need that the truncated differentials in the outbound phase propagate from 8 to 1 active byte through the MixRows transformation, both in the backward and forward direction (see Fig. 51). Since the transition from 8 active bytes to 1 active byte through the MixRows transformation has a probability of about $2^{-56}$, the probability of this part of the outbound phase is $2^{-2.56}=2^{-112}$. Furthermore, to construct a collision at the output (after the feed-forward), the exact value of the input and output difference has to match. Since only one byte is active (see Fig. [5), this can be fulfilled with a probability

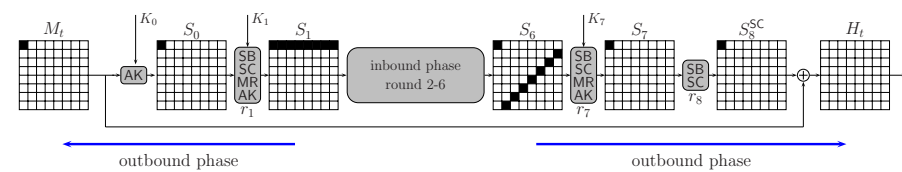

Fig. 5. Differential trail for collision attack on 7.5 rounds 
of $2^{-8}$. Hence, the probability of the outbound phase is $2^{-112} \cdot 2^{-8}=2^{-120}$. In other, words, we have to generate $2^{120}$ starting points (for the outbound phase) in the inbound phase of the attack to find a collision for the compression function of Whirlpool reduced to 7.5 rounds.

Since we can find one starting point with an average complexity of about $2^{64}$ and memory requirements of $2^{8}$, we can find a collision with a complexity of about $2^{120+64}=2^{184}$. The complexity of the attack can be further improved on the cost of higher memory requirements. By using a lookup table with $2^{128}$ entries (generated in a precomputation step), we can find one starting point for the inbound phase with an average complexity of 1 . In other words, we can find a collision for the compression function reduced to 7.5 rounds with a complexity of about $2^{120}$. However, the precomputation step (constructing the lookup table) has a complexity of about $2^{128}$.

Near-Collision for 9.5 Rounds. The collision attack on 7.5 rounds for the compression function can be further extended by adding one round at the beginning and one round at the end of the trail in the outbound phase. The result is a near-collision attack on 9.5 rounds for the compression function of Whirlpool with the following sequence of active bytes:

$$
8 \stackrel{r_{1}}{\longrightarrow} 1 \stackrel{r_{2}}{\longrightarrow} 8 \stackrel{r_{3}}{\longrightarrow} 64 \stackrel{r_{4}}{\longrightarrow} 8 \stackrel{r_{5}}{\longrightarrow} 8 \stackrel{r_{6}}{\longrightarrow} 64 \stackrel{r_{7}}{\longrightarrow} 8 \stackrel{r_{8}}{\longrightarrow} 1 \stackrel{r_{9}}{\longrightarrow} 8 \stackrel{r_{9.5}}{\longrightarrow} 8
$$

Since the 1-byte difference at the beginning and end of the 7.5 round trail will always result in 8 active bytes after one MixRows transformation (see Sect. 3.2), we can go backward 1 round and forward 1 round with no additional cost. Using the feed-forward, the position of two active S-boxes match and cancel each other with a probability of $2^{-16}$. Hence, we get a collision in 50 and 52 bytes for the compression function of Whirlpool with a complexity of about $2^{176}$ and $2^{176+16}=2^{192}$, respectively. With a precomputation step with complexity of $2^{128}$ and similar memory requirement, one can find a near-collision for the compression function of Whirlpool with a complexity of about $2^{112}$ (collision in 50 bytes) and $2^{128}$ (collision in 52 bytes), respectively.

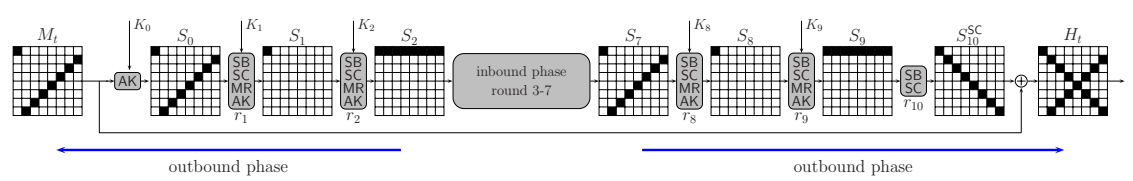

Fig. 6. In the attack on 9.5 rounds we extend the trail one more round at the beginning and at the end of the outbound phase to get a near-collision of Whirlpool

\section{A Subspace Distinguisher for 10 Rounds}

In this section, we present the first cryptanalytic result on the full Whirlpool compression function. The method for extending the previous result on 9.5 rounds is extended to full 10 rounds of the compression function by defining 
a different attack scenario. Instead of aiming for a near-collision, we are interested in distinguishing the Whirlpool compression function from a random function. For this, we will introduce a new kind of distinguishing attack, a so called subspace distinguisher. In the following, $\mathbb{F}_{2}=G F(2)$ always denotes the finite field of order 2 .

For the subspace distinguishing attack, we consider the following problem:

Problem 1. Given a function $f$ mapping to $\mathbb{F}_{2}^{N}$, try to find $t$ input pairs such that the corresponding output differences belong to a vector space of dimension at most $n$ for some $n \leq N$.

Remark. We define Problem [1 in this generic way in order to make it more generally applicable. This will be shown in the extended version of this paper.

\subsection{Solving Problem 1 for the Whirlpool Compression Function}

In this section, we show how the compression function attack described in Sect. 4 can be used to distinguish the full Whirlpool compression function from a random function.

Obviously, the difference between two Whirlpool states can be seen as a vector in the vector space of dimension $N=512$ over $\mathbb{F}_{2}$. The crucial observation is that the attack of Sect. 4 can be interpreted as an algorithm that can find $t$ difference vectors in $\mathbb{F}_{2}^{512}$ (output differences of the compression function) that form a vector space of dimension $n \leq 128$.

To see this, observe that by extending the differential trail from 9.5 to 10 rounds, the 8 active bytes in $S_{10}^{\mathrm{SC}}$ will always result in a fully active state $S_{10}$ due to the properties of the MixRows transformation. Thus the near-collision is destroyed. However, if we look again at Fig. 6] the differences in $M_{t}$ and the differences in $S_{10}^{\mathrm{SC}}$ can be seen as (difference) vectors belonging to subspaces of $\mathbb{F}_{2}^{512}$ of dimension at most 64 .

Even though after the application of MixRows and AddRoundKey the state $S_{10}$ is fully active in terms of truncated differentials, the differences in $S_{10}$ still belong to a subspace of $\mathbb{F}_{2}^{512}$ of dimension at most 64 due to the properties of MixRows. Therefore, after the feed-forward, we can conclude that the differences at the output of the compression function form a subspace of $\mathbb{F}_{2}^{512}$ of dimension $n \leq 128$.

Hence, we can use the attack of Sect. 4 to find $t$ difference vectors forming a vector space of dimension $n \leq 128$ with a complexity of $t \cdot 2^{176}$ or $t \cdot 2^{112}$ using a precomputation step with complexity $2^{128}$. Note that $t \leq 2^{192-112}=2^{80}$ due to the remaining degrees of freedom in the inbound phase of the attack.

Now the main question is for which values of $t$ our attack is more efficient than a generic attack. In other words, how do we have to choose $t$ such that we can distinguish the compression function of Whirlpool from a random function. Therefore, we first have to bound the complexity of the generic attack. This is described in the next section.

\subsection{Solving Problem 1 for a Random Function}

Remarks on the Security Model. In order to discuss generic attack scenarios, we will have to choose a security model. We will adopt the black box 
model introduced by Shannon [23. In this model, a block cipher can be seen as a family of functions parameterized by the secret key $k \in \mathcal{K}$, that is, $E$ : $\{0,1\}^{|k|} \times\{0,1\}^{N} \mapsto\{0,1\}^{N}$, where for each $k \in \mathcal{K}, E_{k}$ is seen as a uniformly chosen random permutation on $\{0,1\}^{N}$.

In [3] it was shown, that an ideal block cipher based hash function in the Miyaguchi-Preneel mode is collision resistant and non-invertible. Based on this, we model our compression function $f$ as black box oracle to which only forward queries are admissible. We also want to note that in all of the following, when we are talking about complexity, we are talking about query complexity. Note that the practical complexity is always greater or equal to the query complexity.

The Generic Approach. In this generic approach the only property used about $f$ is the fact that the outputs of $f$ are contained in the vector space $\mathbb{F}_{2}^{N}$.

Let us now assume that an adversary is making $Q$ queries to the function $f$. Assuming that $Q \ll 2^{N / 2}$, we thus get $K=\left(\begin{array}{c}Q \\ 2\end{array}\right)$ differences $\left(\in \mathbb{F}_{2}^{N}\right)$ coming from these $Q$ queries. For given $n$ and $t \gg n$, we now want to calculate the probability that among these $K$ difference vectors, we have $t$ vectors that span a space of dimension less or equal to $n$.

We will need the following fact about matrices over finite fields. Let $E(t, N, d)$ denote the number of $t \times N$ matrices over $\mathbb{F}_{2}$ that have rank equal to $d$. Then, it is well known ( $c f$. 9] or [13) that

$$
E(t, N, d)=\prod_{i=0}^{d-1}\left(2^{N}-2^{i}\right) \cdot\left(\begin{array}{l}
t \\
d
\end{array}\right)_{2}=\prod_{i=0}^{d-1} \frac{\left(2^{N}-2^{i}\right) \cdot\left(2^{t}-2^{i}\right)}{2^{d}-2^{i}},
$$

where $\left(\begin{array}{l}t \\ d\end{array}\right)_{2}$ denotes the $q$-binomial coefficient with $q=2$.

Proposition 1. Let $n, t, N \in \mathbb{N}$ be given such that $t \gg N>n$. We assume a set of $K$ vectors chosen uniformly at random from $\mathbb{F}_{2}^{N}$. Let $\operatorname{Pr}(K, t, N, n)$ denote the probability that $t$ of these $K$ vectors span a space of dimension not larger than $n$. Then, we have

$$
\begin{aligned}
\operatorname{Pr}(K, t, N, n) & \leq\left(\begin{array}{c}
K \\
t
\end{array}\right) 2^{-t \cdot N} \sum_{d=1}^{n} E(t, N, d) \\
& \leq \frac{1}{\sqrt{2 \pi t}}\left(\frac{K e}{t}\right)^{t} 2^{-(N-n)(t-n)-(n-1)}
\end{aligned}
$$

Proof. Based on the definition of $E(t, N, d)$, it is easy to see that (11) is an upper bound for $\operatorname{Pr}(K, t, N, n)$.

Computing the second bound consists of two steps. Bounding the binomial coefficient and bounding the rest. We get

$$
\begin{aligned}
2^{-t \cdot N} \sum_{d=1}^{n} E(t, N, d) & \leq 2^{-t \cdot N} \cdot 2 \cdot E(t, N, n) \\
& \leq 2^{-t \cdot N+1}\left(\frac{\left(2^{t}-2^{n-1}\right) \cdot\left(2^{N}-2^{n-1}\right)}{2^{n}-2^{n-1}}\right)^{n}
\end{aligned}
$$




$$
\begin{aligned}
& \leq 2^{-t \cdot N+1}\left(2^{n-1} \cdot 2^{t-(n-1)} \cdot 2^{N-(n-1)}\right)^{n} \\
& =2^{-(t-n)(N-n)-(n-1)} .
\end{aligned}
$$

These inequalities are based on two facts. First, it is easy to show that for $t \gg N>n$, we have $E(t, N, n) \leq \sum_{d=1}^{n} E(t, N, d) \leq 2 \cdot E(t, N, n)$. This can be proven by using induction over $n$ and elementary properties of the $q$-binomial coefficient. Second, (14) follows from the fact that the function defined by $f(x)=$ $\left(2^{t}-x\right)\left(2^{N}-x\right) /\left(2^{n}-x\right)$ is strictly increasing on the interval $x \in\left[0,2^{n-1}\right]$.

For the binomial coefficient $\left(\begin{array}{c}K \\ t\end{array}\right)$ we combine the simple estimate $\left(\begin{array}{c}K \\ t\end{array}\right) \leq K^{t} / t$ ! with the following inequality based on Stirling's formula [22]:

$$
\sqrt{2 \pi} t^{t+\frac{1}{2}} e^{-t+\frac{1}{12 t+1}}<t !<\sqrt{2 \pi} t^{t+\frac{1}{2}} e^{-t+\frac{1}{12 t}}
$$

From this we get $\left(\begin{array}{c}K \\ t\end{array}\right) \leq \frac{1}{\sqrt{2 \pi t}}\left(\frac{K \cdot e}{t}\right)^{t}$ and with (16), this proves the proposition.

As a corollary, we can give a lower bound for the number of random vectors needed to fulfill the conditions of the proposition with a certain probability.

Corollary 1. For a given probability $p$ and given $N, n, t$ as in Proposition 1 , the number $K$ of random vectors needed to contain $t$ vectors spanning a space of dimension not larger than $n$ with a probability $p$ is lower bounded by

$$
K \geq \frac{1}{e}(p \sqrt{2 \pi t})^{\frac{1}{t}} \cdot t \cdot 2^{\frac{(N-n)(t-n)+(n-1)}{t}} .
$$

and the number of queries $Q$ to $f$ needed to produce $t$ vectors spanning a space of dimension not larger than $n$ with a probability $p$ is lower bounded by

$$
Q \geq \sqrt{\frac{2}{e}}(p \sqrt{2 \pi t})^{\frac{1}{2 t}} \cdot \sqrt{t} \cdot 2^{\frac{(N-n)(t-n)+(n-1)}{2 t}} .
$$

Proof. Equation (18) follows immediately from (12) and (19) follows from setting $K=\left(\begin{array}{c}Q \\ 2\end{array}\right)=Q(Q-1) / 2$ in (18).

\subsection{Complexity of the Distinguishing Attack}

Table 2 shows the complexities of the generic approach and our dedicated approach for several values of $t$. As can be seen in the table, one can distinguish the full Whirlpool compression function from random with a complexity of about $2^{188}$ with $t=2^{12}$ (or $2^{121}$ with $t=2^{9}$ using a precomputation table). In other words, when performing $2^{188}$ queries to a random function (19) shows that the probability for solving Problem[1 for $t=2^{12}$ is $\ll 1$. To the best of our knowledge this is the first result on the full Whirlpool compression function. 
Table 2. Values for $t, Q$ (query complexity), $C$ (complexity of our attack), and $C_{p}$ (complexity of our attack with precomputation) for $p=1, N=512, n=128$

\begin{tabular}{|c|c|c|c||c|c|c|c|}
\hline $\log _{2}(t)$ & $\log _{2}(Q)$ & $\log _{2}(C)$ & $\log _{2}\left(C_{p}\right)$ & $\log _{2}(t)$ & $\log _{2}(Q)$ & $\log _{2}(C)$ & $\log _{2}\left(C_{p}\right)$ \\
\hline 9 & 148.41 & 185 & 121 & 13 & 195.29 & 189 & 125 \\
10 & 172.84 & 186 & 122 & 14 & 197.28 & 190 & 126 \\
11 & 185.31 & 187 & 123 & 15 & 198.53 & 191 & 127 \\
12 & 191.80 & 188 & 124 & 16 & 199.40 & 192 & 128 \\
\hline
\end{tabular}

\section{Conclusion}

In this paper, we have proposed a new kind of distinguishing attack for cryptanalysis of hash functions. We have successfully attacked the Whirlpool compression function. To the best of our knowledge this is the first attack on full Whirlpool.

We have obtained this result by improving the rebound attack on reduced Whirlpool. First, the inbound phase of the rebound attack was extended by up to two rounds using the available degrees of freedom from the key schedule. This resulted in a near-collision attack on 9.5 rounds of the compression function of Whirlpool. Second, we have shown how to turn this rebound near-collision attack into a distinguishing attack for the full 10 round compression function of Whirlpool.

The idea seems applicable to a wider range of hash function constructions. In particular, the attacks described in this paper can be applied to the hash function Maelstrom [8] in a straight forward manner because of the similarity to Whirlpool (see also [16]). Several SHA-3 candidates are a natural target for this new kind of attack, see for instance 1415. Furthermore, subspace distinguishers can be applied to block ciphers as well. This will be discussed in an extended version of this paper.

\section{Acknowledgements}

The authors wish to thank the anonymous referees for useful comments and discussions. The work in this paper has been supported in part by the European Commission under contract ICT-2007-216646 (ECRYPT II) and in part by the IAP Programme P6/26 BCRYPT of the Belgian State (Belgian Science Policy).

\section{References}

1. Barreto, P.S.L.M., Rijmen, V.: The Whirlpool Hashing Function. Submitted to NESSIE (September 2000), http://www.larc.usp.br/ pbarreto/WhirlpoolPage.html (2008/12/11) (revised May 2003)

2. Biham, E., Shamir, A.: Differential Cryptanalysis of DES-like Cryptosystems. In: Menezes, A., Vanstone, S.A. (eds.) CRYPTO 1990. LNCS, vol. 537, pp. 2-21. Springer, Heidelberg (1991) 
3. Black, J., Rogaway, P., Shrimpton, T.: Black-Box Analysis of the Block-CipherBased Hash-Function Constructions from PGV. In: Yung, M. (ed.) CRYPTO 2002. LNCS, vol. 2442, pp. 320-335. Springer, Heidelberg (2002)

4. Daemen, J., Rijmen, V.: The Wide Trail Design Strategy. In: Honary, B. (ed.) Cryptography and Coding 2001. LNCS, vol. 2260, pp. 222-238. Springer, Heidelberg (2001)

5. Daemen, J., Rijmen, V.: The Design of Rijndael. Information Security and Cryptography. Springer, Heidelberg (2002), ISBN 3-540-42580-2

6. De Cannière, C., Mendel, F., Rechberger, C.: Collisions for 70-Step SHA-1: On the Full Cost of Collision Search. In: Adams, C.M., Miri, A., Wiener, M.J. (eds.) SAC 2007. LNCS, vol. 4876, pp. 56-73. Springer, Heidelberg (2007)

7. De Cannière, C., Rechberger, C.: Finding SHA-1 Characteristics: General Results and Applications. In: Lai, X., Chen, K. (eds.) ASIACRYPT 2006. LNCS, vol. 4284, pp. 1-20. Springer, Heidelberg (2006)

8. Filho, D.G., Barreto, P.S., Rijmen, V.: The Maelstrom-0 hash function. In: SBSeg 2006 (2006)

9. Fisher, S.D.: Classroom Notes: Matrices over a Finite Field. Amer. Math. Monthly 73(6), 639-641 (1966)

10. Knudsen, L.R.: Truncated and Higher Order Differentials. In: Preneel, B. (ed.) FSE 1994. LNCS, vol. 1008, pp. 196-211. Springer, Heidelberg (1995)

11. Knudsen, L.R.: Non-random properties of reduced-round Whirlpool. NESSIE public report, NES/DOC/UIB/WP5/017/1 (2002)

12. Knudsen, L.R., Rijmen, V.: Known-key distinguishers for some block ciphers. In: Kurosawa, K. (ed.) ASIACRYPT 2007. LNCS, vol. 4833, pp. 315-324. Springer, Heidelberg (2007)

13. Lidl, R., Niederreiter, H.: Finite Fields, Encyclopedia of Mathematics and its Applications, 2nd edn., vol. 20. Cambridge University Press, Cambridge (1997); with a foreword by P. M. Cohn

14. Matusiewicz, K., Naya-Plasencia, M., Nikolić, I., Sasaki, Y., Schläffer, M.: Rebound Attack on the Full LANE Compression Function. In: Matsui, M. (ed.) ASIACRYPT 2009. LNCS, vol. 5912, pp. 106-125. Springer, Heidelberg (2009)

15. Mendel, F., Peyrin, T., Rechberger, C., Schläffer, M.: Improved Cryptanalysis of the Reduced Grøstl Compression Function, ECHO Permutation and AES Block Cipher. In: Jacobson Jr., M.J., Rijmen, V., Safavi-Naini, R. (eds.) SAC 2009. LNCS, vol. 5867, pp. 16-35. Springer, Heidelberg (2009)

16. Mendel, F., Rechberger, C., Schläffer, M., Thomsen, S.S.: The Rebound Attack: Cryptanalysis of Reduced Whirlpool and Grøstl. In: Dunkelman, O. (ed.) FSE 2009. LNCS, vol. 5665, pp. 260-276. Springer, Heidelberg (2009)

17. Mendel, F., Rijmen, V.: Cryptanalysis of the Tiger Hash Function. In: Kurosawa, K. (ed.) ASIACRYPT 2007. LNCS, vol. 4833, pp. 536-550. Springer, Heidelberg (2007)

18. Menezes, A.J., van Oorschot, P.C., Vanstone, S.A.: Handbook of Applied Cryptography. CRC Press, Boca Raton (1997), http://www.cacr.math.uwaterloo.ca/hac/

19. National Institute of Standards and Technology: FIPS PUB 197, Advanced Encryption Standard (AES). Federal Information Processing Standards Publication 197, U.S. Department of Commerce (November 2001)

20. NESSIE: New European Schemes for Signatures, Integrity, and Encryption. IST1999-12324, http://cryptonessie.org/

21. Peyrin, T.: Cryptanalysis of Grindahl. In: Kurosawa, K. (ed.) ASIACRYPT 2007. LNCS, vol. 4833, pp. 551-567. Springer, Heidelberg (2007) 
22. Robbins, H.: A remark on Stirling's formula. Amer. Math. Monthly 62, 26-29 (1955)

23. Shannon, C.E.: Communication Theory of Secrecy Systems. Bell Systems Technical Journal 28, 656-715 (1949)

24. Wang, X., Lai, X., Feng, D., Chen, H., Yu, X.: Cryptanalysis of the Hash Functions MD4 and RIPEMD. In: Cramer, R. (ed.) EUROCRYPT 2005. LNCS, vol. 3494, pp. 1-18. Springer, Heidelberg (2005)

25. Wang, X., Yin, Y.L., Yu, H.: Finding Collisions in the Full SHA-1. In: Shoup, V. (ed.) CRYPTO 2005. LNCS, vol. 3621, pp. 17-36. Springer, Heidelberg (2005)

26. Wang, X., Yu, H.: How to Break MD5 and Other Hash Functions. In: Cramer, R. (ed.) EUROCRYPT 2005. LNCS, vol. 3494, pp. 19-35. Springer, Heidelberg (2005)

\section{A Attacks on the Hash Function}

In this section, we present a collision and near-collision for the Whirlpool hash function. The attacks are a straight forward extension of the collision and nearcollision attack on 4.5 and 6.5 rounds of Whirlpool presented in [16]. By adding one round in the inbound phase we can find a collision and a near-collision for Whirlpool reduced to 5.5 and 7.5 rounds, respectively. The core of the attack is a 5 round differential trail, where two fully active states are placed in the middle:

$$
1 \stackrel{r_{1}}{\longrightarrow} 8 \stackrel{r_{2}}{\longrightarrow} 64 \stackrel{r_{3}}{\longrightarrow} 64 \stackrel{r_{4}}{\longrightarrow} 8 \stackrel{r_{5}}{\longrightarrow} 1
$$

Since the outbound phase of the attacks is identical to the previous attacks (see Sect. (4), we only discuss the inbound phase of the attack here (see Fig. 7).

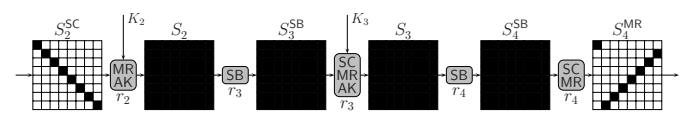

Fig. 7. The inbound phase of the collision attack and near-collision attack on the hash function

It can be summarized as follows.

1. Precomputation: For the S-box, compute a $256 \times 256$ lookup table as described in Sect. 3.2

2. Start with 8 active bytes (differences) at the input of MixRows in round $r_{2}$ $\left(S_{2}^{\mathrm{SC}}\right)$ and propagate forward to the input of SubBytes in round $r_{3}\left(S_{2}\right)$.

3. Start with 8 active bytes at the output of MixRows in round $r_{4}\left(S_{4}^{\mathrm{MR}}\right)$ and propagate backward to the output of SubBytes in round $r_{4}\left(S_{4}^{\mathrm{SB}}\right)$.

4. Next we have to connect the states $S_{2}$ and $S_{4}^{\mathrm{SB}}$ such that the differential trail holds. In other words, we have to find the actual values for $S_{2}$ such that:

$$
\mathrm{SB}\left(\operatorname{MR}\left(\mathrm{SC}\left(\mathrm{SB}\left(S_{2}\right)\right)\right) \oplus K_{3}\right) \oplus \mathrm{SB}\left(\operatorname{MR}\left(\mathrm{SC}\left(\mathrm{SB}\left(S_{2} \oplus \Delta_{1}\right)\right)\right) \oplus K_{3}\right)=\Delta_{2}
$$

where $\Delta_{1}$ denotes the active bytes (differences) in $S_{2}$ and $\Delta_{2}$ denotes the active bytes (differences) in $S_{4}^{\mathrm{SB}}$. In the following, we will show how this equation can be solved with a complexity of about $2^{64}$ by solving the equation for sets of 8 bytes independently. It can be summarized as follows. 
(a) For all $2^{64}$ values of $S_{2}[0,0], S_{2}[1,7], \ldots, S_{2}[7,1]$ compute the first row of $S_{4}^{\mathrm{SB}}$ and check if the above equation holds. Note that due to ShiftColumns, these bytes are shifted to the first row of $S_{3}^{\mathrm{SC}}$ and MixRows works on each row independently. In other words, we get $2^{64}$ candidates for each row of $S_{4}^{\mathrm{SB}}$. Hence, after testing all $2^{64}$ candidates for the first row of $S_{4}^{\mathrm{SB}}$ we expect to find a match for the first row of $\Delta_{2}$.

(b) Do the same for the corresponding 8 bytes for row 2-8 of $S_{4}^{\mathrm{SB}}$.

After testing each set of 8 bytes independently, we will find a state $S_{2}$ such that the differential trail is connected. Finishing this step of the attack has a complexity of about $8 \cdot 2^{64}$ MixRows ( $\approx 2^{64}$ round computations).

Hence, we can compute one starting point for the outbound phase with a complexity of about $2^{64}$. Note that the complexity of the inbound phase can be significantly reduced at the cost of higher memory requirements. By saving $2^{64-s}$ candidates for $S_{4}^{\mathrm{SB}}$ in a list, we can do a standard time/memory tradeoff with a complexity of about $2^{120+s}$ and memory requirements of $2^{64-s}$. By setting $s=0$ we can find $2^{64}$ starting points with a complexity of $2^{64}$ and similar memory requirements of $2^{64}$.

Hence, we can find a collision for Whirlpool reduced to 5.5 rounds with a complexity of about $2^{120}$ and a near-collision for 7.5 rounds in 50 (respectively 52 ) bytes with a complexity of about $2^{120}$ and $2^{112}$ (respectively $2^{128}$ ). All attacks have memory requirements of $2^{64}$. 